\title{
PLANEJAMENTO FINANCEIRO
}

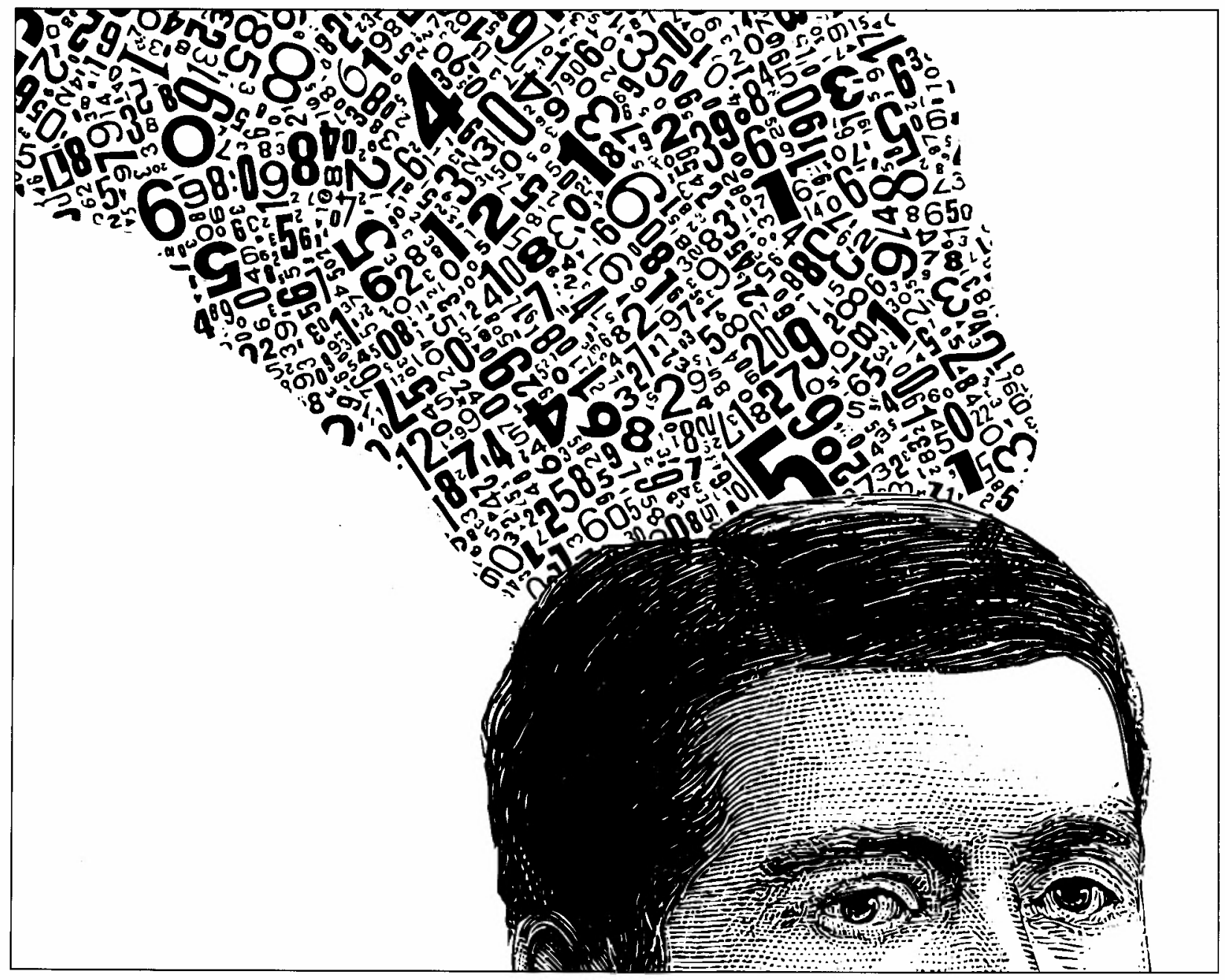

“O objetivo precípuo perseguido numa projeção financeira não é acertar com precisão o valor futuro da variável projetada; antes, o verdadeiro propósito dos instrumentos de análise prospectiva é fornecer elementos válidos de convicção para a tomada de decisão, no momento em que se tem que decidir" ${ }^{*}{ }^{*}$.

\section{- Alexandre Hansen Virzi}

Professor do Departamento de Economia e Administração da UNESP.

\footnotetext{
A nem sempre fácil tarefa do planejamento fiA nanceiro, em que pese o crescente aperfeiçoamento dos métodos probabilísticos e demais técnicas que visam a incorporar o fator externo às
}

projeções, não deve desencorajar o administrador financeiro. No mais das vezes, se a administração evita pormenorizações dos atos, metas, resultados e responsabilidades, bem como a quantificação dos mesmos (aliada a um cronograma), a média gerência, ainda que parcialmente, se incumbe de fazê-lo. A imperiosa realidade do mundo dos negócios não permite somente administrar o cotidiano: antes, é necessário prover de informações, mesmo que incompletas, o tomador de decisões. Não podemos esperar o encerramento dos demonstrativos financeiros para avaliar a performance empresarial. A informação contábil,

$\left.{ }^{*}\right)$ ROXO, Alfredo A. de Oliveira \& ARAÚJO, Marcos Poggi de. (Ver referência bibliográfica número 071.) 
muito embora preciosa, mereceu críticas como: “... muitos modelos incorporam uma visão contábil do mundo, pois eles são projetados para estimar demonstrativos contábeis e, suas equações, naturalmente, incorporam convenções contábeis $e$, conseqüentemente, os modelos não enfatizam as ferramentas de análise financeira tais como: fluxo de caixa, valor presente, risco do mercado etc.". Naturalmente, muito embora a observação tenha procedência, ela é apenas parcialmente real. Isso porque o papel do presente texto é identificar as alternativas para os problemas afetos à administração financeira sem descuidar, todavia, dos aspectos de médio e longo prazo. No nosso entender, escolher e fixar metas não significa uma camisa de força, mas, antes, saber identificar alterações e justificá-las, tornando o processo de planejamento um instrumento hábil e interativo. Senão, vejamos: compreende o sistema de planejamento financeiro a avaliação da origem e da aplicação de recursos - pretérita e prospectivamente. Sempre é,bom lembrar que a origem e a aplicação de recursos, se excluirmos os itens circulantes ${ }^{* 2}$, são equivalentes ao fluxo de caixa (base caixa), em estudos no FASB (Federal
Accounting Standard Boad) sobre a conveniência em "abrir" o fluxo de caixa em: operações, investimentos em ativos e financiamentos. Dentre os modelos de planejamento financeiro que - sem detrimento dos outros - pretendemos destacar, estão:

- Fontes e usos de fundos

- Fluxo de caixa (com incerteza) projetado

- Demonstrações financeiras pro forma.

\section{FONTES E USOS DE RECURSOS: UM EXEMPLO SIMPLIFICADO}

Podemos, a título de exemplo, assumir os demonstrativos financeiros expostos nos quadros I e II, com vistas à elaboração (simplificada) de um quadro de fontes e usos (quadro III).

Observamos, na primeira linha do quadro III,

1. BREALEY, Richard \& MYERS, Stuart. (Ver referência bibliográfica número 078.)

*2. HORNE, James C. V. (Ver referência número 060.)

\section{Quadro I: Balanço e demonstrativo de resultados em moeda forte}

\begin{tabular}{|lrr|}
\hline ATIVO & & \\
\hline & $31 / 12 / \times 8$ & $31 / 12 / \times 9$ \\
caixa e bancos & 400.000 & 620.000 \\
contas a receber & 1.600 .000 & 1.200 .000 \\
estoques & $\underline{2.400 .000}$ & $\underline{3.000 .000}$ \\
ativo circulante & 4.400 .000 & 4.820 .000 \\
Imobilizado técnico & 3.000 .000 & 3.600 .000 \\
dep. acum. & $<1.600 .000>$ & $<1.900 .000>$ \\
ativo permanente & 1.400 .000 & 1.700 .000 \\
total ativo & 5.800 .000 & 6.520 .000 \\
\hline PASSIVO & & \\
\hline & & $31 / 12 / \times 9$ \\
empréstimos & $31 / 12 / \times 8$ & 1.200 .000 \\
contas a pagar & 700.000 & 400.000 \\
outros circulantes & 800.000 & $\underline{400.000}$ \\
Passivo circulante & $\underline{400.000}$ & 2.000 .000 \\
empréstimo longo prazo & 1.900 .000 & 1.400 .000 \\
\hline Patrimônio líquido & $\underline{1.200 .000}$ & $\underline{3.120 .000}$ \\
\hline Capital & $\underline{2.700 .000}$ & 1.400 .000 \\
lucros acumulados & 1.400 .000 & 1.720 .000 \\
total passivo & 1.300 .000 & 6.520 .000 \\
\hline
\end{tabular}


Quadro II: Demonstrativo de Resultados

\begin{tabular}{|lr|}
\hline & $12 / \times 9$ \\
Vendas & 8.000 .000 \\
C.M.V. & 5.000 .000 \\
Lucro Bruto & 3.000 .000 \\
Despesas operacionais & 1.400 .000 \\
Despesas depreciação & 300.000 \\
Despesas financeiras & 440.000 \\
Lucro antes do IR & 860.000 \\
I.R. (35\%) & $\cong$ \\
Lucro líquido & 500.000 \\
Dividendos & 560.000 \\
Lucro exercício & 140.000 \\
& 420.000 \\
\hline
\end{tabular}

as principais fontes advindas do demonstrativo de resultados, bem como os usos (item 1). Logo abaixo, temos os decréscimos (origens/fontes) do ativo (item 2) e os seus acréscimos ou aplicações.Finalmente,temos o mesmo raciocínio para as rubricas do passivo (item 3), gerando um fluxo de fundos de $\$ 1960.000$ entre x8 e x9. Observamos que, a exemplo do que foi colocado pelos professores Hopp e Leite (ver ref. no 009), a idéia de "de onde vem e para onde vai" fica muito mais clara no quadro III do que no tradicional quadro IV, que nos acusa tão somente as variações no CGL.

Para uma abordagem mais completa ver a referência bibliográfica número 083 .

Quadro III: Fonte e uso de fundos simplificados

\begin{tabular}{|c|c|c|c|}
\hline \multicolumn{2}{|l|}{ FONTES } & \multicolumn{2}{|l|}{ USOS } \\
\hline $\begin{array}{l}\text { lucro líquido } \\
\text { depreciação }\end{array}$ & $\begin{array}{l}560.000 \\
300.000\end{array}$ & dividendos & 140.000 \\
\hline contas a receber & 400.000 & $\begin{array}{l}\text { caixa } \\
\text { estoques } \\
\text { imobilizado técnico }\end{array}$ & $\begin{array}{l}220.000 \\
600.000(2) \\
600.000\end{array}$ \\
\hline $\begin{array}{l}\text { empr. curto prazo } \\
\text { empr. longo prazo }\end{array}$ & $\begin{array}{l}500.000 \\
200.000\end{array}$ & contas a pagar & 400.000 \\
\hline Total & 1.960 .000 & Total & 1.960 .000 \\
\hline (1) Demonstrativo & (2) Ativo $\left\{\begin{array}{l}-\mathrm{CP} \\
-\mathrm{LP}\end{array}\right.$ & (3) Passivo & $\begin{array}{l}-C P \\
-L P\end{array}$ \\
\hline
\end{tabular}

Quadro IV: Demonstrativo de Origens e Aplicações (original)

\section{Origens:}

Lucro líquido

(+)depreciações

(+/-)correção monetária

(+/-)equivalência patrimonial

(+/-)variações monetárias

Operações:

dividendos recebidos

dívidas longo prazo

aumentos capital

origens:

aplicacões

aquisição investimento

imobilização

dividendos

aplicações

Variação do CGL
$: 560.000$

: 300.000

: 860.000

$$
\begin{array}{rr}
\mathrm{CGL}_{i} & =2.500 .000 \\
\mathrm{CGL}_{\mathfrak{f}} & =\frac{2.880 .000}{320.000} \\
\text { var } &
\end{array}
$$

$: 200.000$

$\underline{200.000}: 200.000: 1.060 .000$

$: 600.000$

$: 140.000$ 
II.O FLUXO DE CAIXA PROJETADO Quadro V: Fluxo de Caixa (Cash Flow)

\begin{tabular}{|c|c|c|c|c|c|c|c|}
\hline ENTRADAS & nov. & dez. & jan. & fev. & mar. & abr. & mai. \\
\hline à vista & 40.000 & 80.000 & 80.000 & 60.000 & 40.000 & & \\
\hline 30 dias & & 280.000 & 560.000 & 560.000 & 420.000 & 280.000 & \\
\hline 60 dias & & & 80.000 & 160.000 & 160.000 & 120.000 & 80.000 \\
\hline total entradas & 40.000 & 360.000 & 720.000 & 780.000 & 620.000 & 400.000 & 80.000 \\
\hline saídas & & . & & & & & \\
\hline à vista & 24.000 & 48.000 & 48.000 & 36.000 & 24.000 & & \\
\hline 30 dias & & 168.000 & 336.000 & 336.000 & 252.000 & 168.000 & \\
\hline 60 dias & & & 48.000 & 96.000 & 96.000 & 72.000 & 48.000 \\
\hline máquina & & & 800.000 & & & & \\
\hline aluguel & 15.000 & 15.000 & 15.000 & 15.000 & 15.000 & 15.000 & 15.000 \\
\hline salários & 16.000 & 16.000 & 16.000 & 16.000 & 16.000 & 16.000 & 16.000 \\
\hline empréstimo & & & & 460.000 & & & \\
\hline saídas & & & 1.263 .000 & 959.000 & 403.000 & & \\
\hline
\end{tabular}

A empresa planeja o seu fluxo de caixa para o próximo trimestre. As vendas dos últimos dois meses foram $\$ 400.000$ e $\$ 800.000$ e as vendas previstas para os próximos três meses são de $\$ 800.000, \$ 600.000$ e $\$ 400.000$. As vendas à vista são da ordem de $10 \%, 70 \%$ é vendido para pagamento em 30 dias e $20 \%$ para 60 dias. As compras da empresa representam $60 \%$ das vendas, sendo o esquema de pagamentos como segue: $10 \%$ à vista, $70 \%$ em 30 dias, 20\% em 60 dias. Em janeiro, será comprada uma máquina por $\$ 800.000$, à vista. Em fevereiro serão pagos empréstimos totalizando $\$ 460.000$. Aluguéis mensais de $\$ 15.000$ e salários de $\$ 16.000$. Ajudemos a montar o fluxo para o gerente financeiro, conforme o quadro $\mathrm{V}$.

Em que pese a exagerada simplificação do exemplo, encontramos o artigo de Eugene $M$. Lerner, "Simulating a Cash Budget" (ref. no 084), e muitos outros trabalhos, aliando ao problema formulações estocásticas mais adequadas à abordagem na nossa conjuntura, mas que fogem ao escopo do presente texto.
Quadro VI: Resumo

\begin{tabular}{|c|c|c|}
\hline $\begin{array}{l}\begin{array}{l}\text { Saldo inicial janeiro (balanço) } \\
+ \text { entradas } \\
\text { - saídas }\end{array} \\
\text { saldo final janeiro }\end{array}$ & $\stackrel{+}{(-)}$ & $\begin{array}{r}620.000 \\
720.000 \\
1.263 .000 \\
77.000\end{array}$ \\
\hline 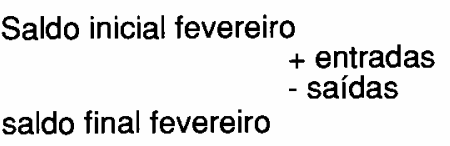 & - & $\begin{array}{r}77.000 \\
780.000 \\
959.000 \\
(102.000)\end{array}$ \\
\hline $\begin{array}{l}\text { Saldo inicial março } \\
\text { saldo final }\end{array}$ & & $\begin{array}{r}(102.000) \\
620.000 \\
403.000 \\
115.000\end{array}$ \\
\hline
\end{tabular}

\section{DEMONSTRAÇÕES FINANCEIRAS} PRO FORMA

O planejamento financeiro tem como input inicial para a elaboração dos diversos orçamentos que compõem as demonstrações financeiras "pro forma" o orçamento de vendas. Sugerimos a seguir uma metodologia simples para cenários alternativos e teceremos um breve comentário sobre os itens 
operacionais que variam teoricamente com vendas.

Existem vários modelos estocásticos de previsão de vendas. No nosso caso, pretendemos determinar qual o poder de previsão que um dado teste pode nos dar quando relacionamos a verba despendida em propaganda $\mathrm{e}$ as vendas relacionadas à verba gasta.

Suponha que queremos usar os seguintes dados coletados em dois anos:

\section{Tabela 1: Vendas $\mathrm{x}$ promoção}

\begin{tabular}{|lrrrrrrrr|}
\hline trimestre & 1 & 2 & 3 & 4 & 5 & 6 & 7 & 8 \\
(y) vendas & & & & & & & & \\
(10000/u) & 25 & 13 & 8 & 20 & 25 & 12 & 10 & 15 \\
(x) promoção & 11 & 5 & 3 & 9 & 12 & 6 & 5 & 9 \\
\hline
\end{tabular}

Desejamos construir um intervalo de confiança para uma futura observação de $Y$ dado um determinado $X$. A reta de estimação é:

$\hat{y}=a+b x=\bar{y}+b(x-\bar{x})$ com desvio de precisão $y-\hat{y}$

sendo $E(Y-\hat{y})=0$

$\operatorname{VAR}(Y-\hat{y})=\operatorname{VAR}(Y)+\operatorname{VAR}(\hat{y})=$

$$
\sigma \mathrm{e}^{2}+\frac{\sigma \mathrm{e}^{2}}{\mathrm{n}}+\sigma \mathrm{e}^{2} \frac{(\mathrm{x}-\overline{\mathrm{x}})^{2}}{\sum(\mathrm{x}-\overline{\mathrm{x}})^{2}}
$$

Como é amostral: (troco $\sigma \mathrm{e}^{2}$ por $\mathrm{Se}^{2}$ )

Ic $(Y(x), 1-\alpha)=\hat{y} \pm \frac{\tau \alpha}{2}(n-2) \operatorname{Se} \sqrt{1+\frac{1}{n}+\frac{(x-\bar{x})^{2}}{\sum\left(x_{i}-\bar{x}\right)^{2}}}$

Para o modelo $\hat{y}=a+b x$, temos genericamente:

\section{Tabela 2: ANOVA}

\begin{tabular}{|c|c|c|c|c|}
\hline Fonte & $\begin{array}{l}\text { Graus de } \\
\text { Liberdade }\end{array}$ & $\begin{array}{l}\text { Soma dos } \\
\text { Quadrados }\end{array}$ & $\begin{array}{l}\text { Quadrado } \\
\text { médio }\end{array}$ & $F$ \\
\hline $\begin{array}{l}\text { regressão } \\
\text { resíduo }\end{array}$ & $\begin{array}{c}1 \\
n-2\end{array}$ & $\begin{array}{l}\text { SQ Reg } \\
\text { SQ Res }\end{array}$ & $\begin{array}{c}\text { (SQ reg : 1) = } \\
\text { QM Reg } \\
\text { [SQ Res : }(n-2)] \\
=\mathrm{Se}^{2}\end{array}$ & $\begin{array}{c}\text { QMReg: } \mathrm{Se}^{2} \\
\mathrm{R}^{2}\end{array}$ \\
\hline Total & $n-1$ & SQ Tot & $\begin{array}{c}\text { [SQ Tot: }(n-1)] \\
=\mathrm{S}^{2}\end{array}$ & \\
\hline
\end{tabular}

Fonte: ver a referência número 056 .

Sendo o poder de explicação do teste dado por $\mathrm{R}^{2}=\frac{\mathrm{SQ} \text { REG }}{\mathrm{SQ} \text { TOT }}$;

sendo F a estatística FISHER - SNEDECOR; acha-se da tabela 1: $\quad \sum y=128 \quad \sum x=60$ $\sum y^{2}=2352 \quad \sum x^{2}=522 \quad \sum x y=1101$.

$1^{\circ}$ passo: o coeficiente angular da reta $-\beta$ $\beta=\frac{\sum x y-n \cdot \bar{x} \cdot \bar{y}}{\sum x^{2}-n(\bar{x})^{2}}$ substituindo $\frac{1101-8 \cdot 7,5 \cdot 16}{522-8(7,5)^{2}}=1,96$

mas

$$
\mathrm{a}=\overline{\mathrm{y}}-\mathrm{b} \bar{x} \text { então } \mathrm{a}=16-(1,96) 7,5 \therefore \mathrm{a}=1,31
$$

logo

$\hat{y}=1,31+1,96 x$

SQ TOT $=\mathrm{S}^{2} \mathrm{y}(\mathrm{n}-1)$ ou $\sum(\mathrm{y}-\overline{\mathrm{y}})^{2}=$

$(25-16)^{2}+\ldots(15-16)^{2}=304$

SQ Res $=\Sigma(\mathrm{y}-\hat{\mathrm{y}})^{2}$ ie, substituo na reta $\hat{y}=1,31+1,96 x$

$\begin{array}{lllllllll}\bar{x} & 11 & 5 & 3 & 9 & 12 & 6 & 5 & 9\end{array}$

$\begin{array}{lllllllll}\bar{y} & 25 & 13 & 8 & 20 & 25 & 12 & 10 & 15\end{array}$

$\begin{array}{lllllllll}\hat{y} & 22,87 & 11,11 & 7,19 & 18,95 & 24,84 & 13,07 & 11,11 & 18,95\end{array}$

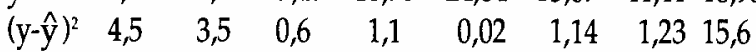

$\sum(\mathrm{y}-\hat{\mathrm{y}})=27,5$

SQ Reg $=b^{2}\left[\sum x^{2}-\frac{\left(\sum x\right)^{2}}{n}\right]=(1,96)^{2}\left[522-\frac{(60)^{2}}{8}\right]=276,59$

Aplicando-se à tabela 1 os dados da tabela 2, tem-se a tabela 3:

\section{Tabela 3: ANOVA Aplicada}

\begin{tabular}{|c|c|c|c|}
\hline Graus de lib. & $\begin{array}{c}\text { Soma dos } \\
\text { Quadrados }\end{array}$ & $\begin{array}{c}\text { Quadrado } \\
\text { Médio }\end{array}$ & $\begin{array}{c}\text { Estatistica } \\
\text { Reg 1 (1) }\end{array}$ \\
276,5 & 276,5 & $\mathrm{~F}=\frac{276}{4,5}=60,33$ \\
Res 6 (8-2) & 27,5 & 4,58 & \\
Total 7 (8-1) & 304 & 48,43 & $\mathrm{R}^{2}=\frac{276}{304}=0,908$ \\
\hline
\end{tabular}

Assim, desde que o modelo é explicativo $\left(\mathrm{R}^{2}=\right.$ $90,8 \%$ ), aplicaremos aos dados a fórmula de intervalo de confiança abaixo:

$\operatorname{IC~}(\operatorname{IP} \hat{y}(x) ; 1-\alpha)=\left(a+b x_{0}\right) \pm \tau \gamma, n-2 . \operatorname{Se} \sqrt{1+\frac{1}{N}+\frac{\left(x_{0}-\bar{x}\right)^{2}}{\sum\left(x_{i}-\bar{x}\right)^{2}}}$

Da tabela de DISTRIBUIÇÃO de Student,

$\frac{\tau \alpha}{2}(n-2)$ sendo $\gamma=6$ e $\alpha=5 \%$, obtemos 2,447 .

$\mathrm{Se}^{2}=[\mathrm{SQ}$ Res $:(\mathrm{n}-2)]=\mathrm{Se}^{2}=4,58$ (ANOVA aplicada) $\therefore$

$\mathrm{Se}=\sqrt{4,58}=2,14$

$\sum\left(x_{i}-\bar{x}\right)=72$. 
Substituindo na fórmula acima:

$\operatorname{IP}(\hat{y}(n): 1-\alpha)=(1,315+1,9588.8) \pm 2,447.2,14: \sqrt{1+\frac{1}{8}+\frac{(8-7,5)^{2}}{72}}$

IP $=16,979 \pm 5,56$ ou seja: eu tenho $95 \%$ de confiança de que gastando 8 ( $x 0)$ de promoção, as minhas vendas estarão entre:

$$
\text { ] 11,4 e } 22,53 \text { [ }
$$

Supondo, então, que as minhas vendas no próximo exercício possam estar entre 11 e $22 \mathrm{mi}$ lhões - em moeda constante - eu facilmente poderia projetar as minhas demonstrações financeiras pro forma. Veja a tabela 4 como exemplo.

\section{Tabela 4: Demonstrativo de Resultado}

\begin{tabular}{|c|c|c|c|c|}
\hline & $\mathrm{x} 9$ & x10 pessimista & x10 moderado & x10 otimista \\
\hline 1. vendas & 8.000 .000 & 11.000 .000 & 16.500 .000 & 22.000 .000 \\
\hline 2. CMV & 5.000 .000 & 6.500 .000 & 9.250 .000 & 12.000 .000 \\
\hline 3. dup. & 300.000 & 300.000 & 300.000 & 300.000 \\
\hline 4. operac. & 1.400 .000 & 2.280 .000 & 2.720 .000 & 3.160 .000 \\
\hline 5. financ. & 440.000 & 440.000 & 440.000 & 440.000 \\
\hline 6. LAIR & 860.000 & 1.480 .000 & 3.790 .000 & 6.100 .000 \\
\hline 7. IR & 300.000 & 518.000 & 1.326 .500 & 2.135 .000 \\
\hline 8. LL & 560.000 & 962.000 & 2.463 .500 & 3.965 .000 \\
\hline 9. DIV & 140.000 & 192.500 & 288.750 & 385.000 \\
\hline 10. L. exerc. & 420.000 & 769.500 & 2.174 .750 & 3.580 .000 \\
\hline
\end{tabular}

Assumindo-se que:

1) temos duas opções viáveis e uma terceira moderada;

2) que cerca de $1 / 5$ do CMV corresponde aos CIF fixos; assim, ele representa em $\times 0=62,5 \%$ e em $\times 10$ somente $50 \%$ ( $80 \%$ de 62,5 sãovariáveis),

$\mathrm{x} 10 \mathrm{CIF}$ - pessimista $=1.000 .000+5.500 .000(\mathrm{CV})$ $=6.500 .000(59,09 \%$ de vendas $)$

$\mathrm{x} 10 \mathrm{CIF}$ - moderado $=(\mathrm{CF}) 1.000 .000+$ $8.250 .000(\mathrm{CV})=9.250 .000(56,6 \%$ de vendas $)$

$\mathrm{x} 10 \mathrm{CIF}$ - otimista $=(\mathrm{CF}) 1.000 .000+11.000 .000(\mathrm{CV})$ $=12.000 .000$ ( $54,5 \%$ de vendas)

3) como a depreciação é fixa, ela se mantém não há necessidade de nova maquinaria;

4) eu aumentarei os meus gastos com propaganda em $8 \%$ das vendas, logo: $x 10$ pessimista $-1.400 .000+880.000=2.280 .000$

x10 moderado $-1.400 .000+1.320 .000=2.720 .000$ x10 otimista $\quad-1.400 .000+1.760 .000=3.160 .000$; as despesas administrativas são fixas e as tributárias, semi-fixas, não cabendo aqui maiores detalhamentos;

5) as despesas financeiras - se o meu esforço mercadológico for calcado em créditos espontâneos (fornecedores, despesas, provisionadas etc.) - são fixas;
6) LAIR;

7) IR = alíquota é de 35\%;

8) L Líquido;

9) a política de dividendos da firma pertence a uma abordagem mais específica que foge ao escopo do trabalho, propomos aqui que varie em percentual das receitas de vendas, ie, $1,75 \%$ de 8.000.000 é equivalente a 140.000 .

10) as vendas estão em três patamares de variação e os lucros, idem, como podemos ver abaixo:

$\begin{array}{rlll}x 10 \mathrm{P} & \times 10 \mathrm{M} & \times 10 \mathrm{O}\end{array}$

crescimento \% vendas (base $\mathrm{x} 9$ ) $\quad 37,5 \quad 106,25 \quad 175$ crescimento \% lucro exercício (base x9) 83,2 417,79 752,4;

evidencia-se o efeito da alavancagem combinada (operacional e financeira).

\section{Demonstrações financeiras pro forma - Observações finais}

A decisão orçamentária não é, efetivamente, algo simples de ser resolvido porquanto para cada tipo de orçamento obtemos "vieses" que, todavia, não descaracterizam o objetivo-chave do mesmo: planos e metas quantificados com atribuição de responsabilidade para cada centro.

No que diz respeito às técnicas orçamentárias, temos dois grandes grupos de variáveis de escolha: 


\section{A - Com relação à moeda:}

1- Orçamento em moeda estável - US\$, BTN etc.

2- Orçamento em moeda instável - reajustandose com inflação.

3- Orçamento em moeda corrente - necessitando examinar cada item durante o período a ser orçado. Exemplo:

- origem interna: CIP, tendências sazonais, reajustes periódicos etc.

- origem externa: taxa cambial

- mão de obra: reajustes salariais, turnos de trabalho etc.

- depreciação: reajuste de acordo com indexador.

- outros custos: desagregação item por item dos componentes e especificação de variação: preço, eficiência mista.

- correção monetária: afeta o demonstrativo, depreciação etc.

- definição do preço de venda: política de fixação, mark-up.

4- Moeda Forte - conversão para moeda estável, como propõe a resolução CVM 64 e regulamentada em 89 para a BTN ou procedimentos como o FASB 8 ou o 52 .

O orçamento em moeda forte ficará igual ao de moeda estável, mas o acompanhamento orçamentário poderá informar as causas das variações se de preço, eficiência, misto, quantidade... Sugere-se, assim, a utilização do orçamento em moeda corrente. Para este último, é particularmente interessante o trabalho de Alexandre Assaf Netto (ver referência 074).

B - Com relação à forma de elaboração do orçamento:

1- Orçamento tradicional

2- Orçamento programa - utilizado inicialmente pela GM, Du Pont e depois na esfera governamental (ver Pyhrr, referência 094, que divide em função, programa, subprograma, projeto, atividade).

3- Base Zero: a cada período, redefinem-se os planos e justificam-se as dotações orçamentárias (ver Pyhrr, referência 094).

Finalmente, dado que o objetivo do presente texto não é o de ser exaustivo, mas, antes, de nortear o leitor para as vertentes atuais e alternativas válidas, cumpre lembrar que o hábito atualmente difundido de usar somente orçamentos em moeda forte ou constante ocasiona vários "bias" como por exemplo: o orçamento quando "traduzido" ou passado para um único indexador/índice, certamente ocasionará distorções dado que vários de seus compromissos estão atrelados a outros indicadores. Citemos: salários - ao IPC com um plus de 5\% se for mínimo, a cada trimestre. Insumos - à taxa cambial, se importados. Energia - aos reajustes especiais de tarifação visando à recomposição da Fair Return etc. Essas distorções não poderão ser imputadas à não consecução orçamentária e/ou a problemas de eficiência. Se flexibilizarmos o orçamento, os efeitos inflacionários tampouco estarão sendo captados no material direto, ou seja: presume-se que no preço padrão já estaria "embutido" o efeito inflacionário. Existem, assim, ex-ante, componentes exógenos ao orçamento que prejudicam a análise de eficiência. Uma possível sugestão seria planejar o padrão como corrente e de acordo com o índice apropriado ao item. Mudanças exógenas seriam remanejadas no bojo do orçamento original, alterando-se o item facilmente, desagregando-se o que não é variação de eficiência. Quanto ao problema de se planejar, calculando a base de inflação própria da empresa, contornando inclusive o problema de base de preço a ser usado nas projeções (se de início ou fim do exercício), temos a excelente digressão de Famá (ver ref. $\mathrm{n}^{\mathrm{o}}$ 037) que nos sugere usar a raiz quadrada dos índices de Layespere e Paache. Finalmente, esperamos ter contribuído aos interessados na matéria com sinalizações mais seguras e/ou práticas alternativas que venham a prestar confiabilidade ao planejamento financeiro. Ao leitor desejo, invejosamente para com a bibliografia recomendada, "bom apetite".

\section{REFERÊNCIAS BIBLIOGRÁFICAS}

Heraldo Vasconcellos

Bibliotecário, chefe do Serviço de Documentação da Biblioteca Karl A. Boedecker da EAESP/FGV.

\section{Observações}

A pesquisa foi realizada no acervo da Biblioteca da EAESP/FGV; as referências bibliográficas foram organizadas em ordem alfabética, dentro de cada ano de publicação (os anos aparecem em ordem cronológica decrescente). As referências precedidas de asterisco estão disponíveis no acervo da Biblioteca. 
1989

001. ALTANY, D.R. "The stretch-limo business cycle: is the expansion's end finally in sight?" Industry Week, 238:74-5, May 151989.

002. "BEFORE you leap, look at the numbers that count". Business Week:187-90+, Dec. 26/Jan. 2 $1988 / 89$.

003. BEST, B. "What's new in prospective financial information". The CPA Journal, 59:50-3, Aug. 1989.

004. BRINNER, R.E. \& WINSBY, R. "The evolution of the business of business economics". Business Economics, 24:32-4, Apr. 1989.

005. *CAMINITI, S. "Winners for slow times". Fortune, 119(2):47-9, Jan. 161989.

006. COOPER, J.C. \& MADIGAN, K. "The expansion has at least a year of life left 1989". Business Week: 102-4, Dec. 26/Jan. 2 1988/1989.

007. HAX, A.C. "Building the firm of the future". Sloan Management Review, 30:75-82, Spring 1989.

008.*HOPP, João Carlos \& LEITE, Hélio de Paula. "O mito da liquidez". Revista de Administração de Empresas, 29(4):63-9, out./dez. 1989.

009. HUTTON, C. "Who will do well?" Fortune, 120:67-70, July 171989.

010. KINDEL, S. "The great companies of the 90s". Financial World, 158:32-7, Feb. 211989.

011. KOLINS, W. "Questions and answers on perspective financial settlements". The Practical Accountant, 22:80+, Sept. 1989.

012."LACZNIAK, E.R. "A tough future for Australian business?" Long Range Planning, 22(1):48-60, Feb. 1989.

013. MAGNET, M. "Stars of the 1980's cast their light". Fortune, 120:66-76, July 31989.
014. MAKRIDAKIS, S. "Management in the 21st century". Long Range Planning, 22(2):37-53, Apr. 1989.

015. "MANAGING for the global decade". Business Month, 134:26-71,July 1989.

016. POPOVICH, L. "Bush confronts the 101st: what's ahead for industry?" Forest Industries, 116:22-3, Jan. 1989.

017. QUICKEL, S.W. "High anxiety". Business Month, 133:36-51, Jan. 1989.

018. " $\mathrm{R} \& \mathrm{D}$ spending growth continues to slow". Research Technology Management, 32:3, May/June 1989.

019. ROBINSON, D.G. "The 1990s: from managing to leading". Supervisory Management, 34:510, June 1989.

020. "THE YEAR of the manager: the fourth annual readers forecast". Canadian Business, 62:2531, Jan. 1989.

\section{8}

021. ALTANY, D.R. "What's ahead for 89". Industry Week, 237:29+, Nov. 211989.

022. BAGBY, J.W. "Management discussion of business performance: on analytical and empirical evaluation". American Business Law Journal, 26:57-98, Spring 1988.

023. "CLASSIC packaging design will prevail in the next century". Marketing News, 22:21, Sept. 261988.

024. DENNIS, D.L. "What the forecast means for 16 industries". Fortune, 117:81-2+, Jan. 181988.

025. ${ }^{*} D R U C K E R$, P.F. "The coming of the new organization". Harvard Business Review, 66(1):4553, Jan./Feb. 1988.

026. EVANS, M.K. "Investment sights set low for the 90s". Industry Week, 236:84, Apr. 181988. 
027. "OUTLOOKS for NACM industries". Business Credit, 90:29, Oct. 1988.

028. "RECOVERY holds steady course. OEM output will continue to expand through $88^{\prime \prime}$. Industrial Distribution, 77:167, Jan. 1988.

029. SAMPLE, J.O. "Surveys that mean business". Association Management, 40:24-9, July 1988.

030. "VIEWS from the top: what is the most significant challenge American business faces?" Business Month, 132:66-8+, July/Aug. 1988.

031. WATTENBERG, B.J. "Their deepest concerns". Business Month, 131:26-9+, Jan. 1988.

032. WOOD, W.C. "Strong industrial upturn in 88". Industrial Distribution, 77:69, Feb. 1988.

1987

033. CAMERON, A. \& CHAPMAN, G. "Providing forecasts and projections: a guide to the complex new rules". The Practical Accountant, 20: 94-8+, Aug. 1987.

034. DIETZEL, J. "1988 forecast: no booms or busts are on the horizon". Plastics World, 45:41924, Dec. 1987.

035. "EARNINGS outlook: how to play along with T. Boone, Sir Jimmy, and the gang". Business Week, 140:4+, Dec. 29/Jan. 51987.

036. "ED on future-oriented financial statements released". CA Magazine, 120:14-15, Mar. 1987.

037. FAMÁ, Rubens. Análise do desempenho operacional das empresas com a utilização de númerosindices. São Paulo, FEA/USP, 1987. (Tese de Doutorado)

038. "FORECAST 1987". Financial World, 156:22-57, Jan. 61987.

039. JONES, A. "The crystal ball". Financial World, 156:21, Apr. 71987.
040. ${ }^{*}$ KOENIG, P. "After the crash of 1989". Euromoney, :115+, Sept. 1987.

041. MAY, T. "Higher profits lie ahead". Fortune, 116:127-8+, Fall 1987.

042.*__. "Surprise: faster growth lies ahead". Fortune, 115: 52-7, Jan. 191987.

43. MENZIES, P.T. "Financial control as an aid to management". Management Decision, 25(2): 54$8,1987$.

044. MURRAY, T.J. "John Sculley looks ahead again". Business Month, 130:30-2, Oct. 1987.

45.*NIELSEN, J. "What the forecast means for 16 industries". Fortune, 115(2):57-9, Jan. 191987.

046.*PERSONICK, V.A. "Industry output and employment through the end of the century". Monthly Labor Review, 110(9):30-45, Sept. 1987.

047. ${ }^{* \prime}$ QS \& AS from the AICPA technical hotline". Journal of Accountancy, 163:72+, Apr. 1987.

048. RICH, S.R. \& GUMPERT, D. "Closely watched trends". Inc., 9:94, Jan. 1987.

049." SEVEN wary views from the top". Fortune, 115(3):48-53, Feb. 21987.

050. SLOAN, J. "Two business forecasts: the good and the bad". Association Management, 39:412, Jan. 1987.

051. SOMMER, D.W. "Business outlook: big isn't so beautiful". Industry Week, 233:22, Apr. 1987.

052. TEMPLE, R.M. "Plain paper prospective financial statements". The CPA Journal, 57:81-2, July 1987.

053. "WHAT the experts say". Nation's Business, 75:26-7, Jan. 1987.

054. WINGO, W. "What's ahead for tomorrow". Nation's Business, 75: 68+, Sept. 1987. 
1986

055. BERLINER, R.W. "Improving the crystal ball, new standards for CPA services, procedures and reports". Financial Management, 88:25-7, Sept. 1986.

056.*BUSSAB, W.O. Análise de variância e regressão. São Paulo, Atual, 1986, 147p.

057. CLOUSER, E.R. "The oracles ol the Delphi panel". Risk Management, 33:56-8+, Nov. 1986.

058. COOK, R. "Plans for a better future". Computer Decisions, 18: 60+, Sept. 301986.

059. FORSYTH, R.W. “Boom bust or muddle? Here's what two leading Wall Street economists think". Barron's, 66:6-7+, Dec. 1986.

060. HORNE, James C.V. Fundamentals of financial management. Englewood Cliffs, Prentice-Hall, 1986, 731p., p. 159-60.

061. HORTON, T.R. "Are you helping to shape America's future?" Security Management, 30:110+, Nov. 1986.

062."LOUREIRO, José Carlos. Modelo para planejamento financeiro em ambientes inflacionários. São Paulo, EAESP/FGV, 1986. (Dissertação de Mestrado.)

063. NAGAN, P.S. "Ho-hum game awaits 4th quarter action". ABA Bank Journal, 78:12+, Dec. 1986.

064. TALSON, P. "Looking ahead to forecasting". Supervision, 48: 6-8, Dec. 1986.

065." REMEMBER steam cars and plastic teeth?" The Economist, 300:71, Sept. 131986.

066. *SILVA, Roberto Alves e. Lucro: um estudo do planejamento e controle no contexto inflacionário. São Paulo, EAESP/FGV. 1986. (Dissertação de Mestrado.)

067. SOMMER, D.W. "What CEOs see ahead: faint hopes for $87^{\prime \prime}$. Industry Week, 231:45-8, Nov. 241986.

068. WEIDENBAUM, M.L. "The Business landscape in 2001". Across the Board, 23:60-4, May 1986.

069. "YEAR ahead looks pretty good-except for steel". Industry Week, 228:18+, Jan. 1986.

\section{5}

070. "CEO report: strategies for the 1990s". Purchasing, 98:69-107, Jan. 311985.

071. ROXO, Alfredo A. de Oliveira \& ARAÚJO, Marcos Poggi de. Planejamento e gestão empresarial sob inflação - Um método geral para tratamento das projeções financeiras. Rio de Janeiro, Campus, 1985.

072.*SCHUBERT, Pedro. Orçamento empresarial integrado. Rio de Janeiro, Livros Técnicos e Científicos, 1985, 429p.

\section{4}

073. DANOS, P. "Band reters' use of management financial forecasts: experiment in expert judgement". The Accounting Review, 59:547-73, Oct. 1984.

\section{3}

074. ASSAF NETO, Alexandre. Aspectos da projeção de resultados e análise do preço de venda e ponto de equilibrio de empresas inseridas em contexto inflacionário - o caso brasileiro. São Paulo, FEA/USP, 1983. (Tese de Doutoramento)

075. *BRULL, Thomas Daniel. Planejamento do lucro: um modelo integrado de simulação que incorpora o impacto inflacionário. São Paulo, USP, 1983, 174p. (Dissertação de Mestrado)

\section{2}

076. *BOUCINHAS, José Fernando da Costa. A aplicação de modelos ao proceśso de planejamento na empresa. São Paulo, USP, 1982, 153p. (Tese de Doutoramento) 
077. *OLIVEIRA, Arnaldo Prestes. O desenvolvimento da análise financeira nos últimos anos. São Paulo, EAESP/FGV, 1982. (Dissertação de Mestrado.)

1981

078. BREALEY, Richard \& MYERS, Stuart. Principles of corporate finance. New York, McGrawHill, 1981, 794p, p.644-5.

079. CARRARO, F. Mauro. O planejamento financeiro de projetos em condições de inflação. São Paulo, FEA/USP, 1981.

080. HELFERT, Erich A. Techniques of financial analysis. $5^{\mathrm{a}}$ ed. Homewood, Irwin, 1981.

081. SANTOS, Celso da Costa. Práticas de planejamento econômico-financeiro. São Paulo, FEA/ USP, 1981, 136p. (Dissertação de Mestrado)

082. *WALTER, Milton Augusto. Orçamento integrado. São Paulo, Saraiva, 1981. 103p.

1980

083. *DE SALVO, A. "Cash Management converts dollars into working assets". In: SMITH, K.V. Readings on the management of working capital. $2^{\mathrm{a}}$ ed., St. Paul, West Publishing, 1980, 624 p.

084. *LERNER, Eugene M. "Simulating a Cash Budget". In: SMITH, K.V. Readings on the Management of Working Capital. $2^{\mathbf{a}}$ ed., St. Paul, West Publishing, 1980, 624 p.

1979

085. FECHIO, Lino Eduardo Real. Esquema geral de planejamento e controle financeiro integrado: a experiência de implantação em um projeto binacional. São Paulo, FEA/USP, 1979. (Dissertação de Mestrado)

1978

086. JONES, Reginald L. Orçamento: a chave do planejamento e controle. São Paulo, McGrawHill, 1978. 296p.

087. MOREIRA, José Carlos. Controle financeiro. São Paulo Saraiva, 1978. 277p.

1977

088. ${ }^{*}$ CHEEK, Logan M. Zero-base budgeting comes of age: what it is and what it takes to make it work. New York, AMACOM, 1977. 314p.

089.*STEDILE, Wanderlei Ivan. Planejamento financeiro e inflação. São Paulo, EAESP/FGV, 1977, 112p. (Dissertação de Mestrado)

1976

090. FINANCIAL ACCOUNTING STANDARDS BOARD. Scope and implications of the conceptual framework project. Stanford, 1976, 24p.

091. HUNT, Lacy H. Dynamics of forecasting financial cycles theory, technique and implementation. Greenwish, Jai, 1976, 296p.

1974

092. FONSECA, Jairo Simon da. Contribuição ao emprego de modelos de regressão no processo de previsão. São Paulo, FEA/USP, 1974. (Tese de Livre Docência)

093. ${ }^{*}$ LEV, Baruch. Financial statement analysis: a new approach. Englewood Cliffs, Prentice-Hall. 1974, 262p.

1973

094.*PYHRR, Peter A. Zero-base budgeting: a practical management tool for evaluating expenses. New York, Wiley, 1973, 231p.

\section{1}

095.*BYRNE, R.F. Studies in budgeting. Amsterdam, North-Holland. 1971. 
096. ${ }^{*}$ CHAMBERS, John C. et alii. "How to choose the right forecasting technique". Harvard Business Review, 49(4):45-74, July/Aug. 1971.

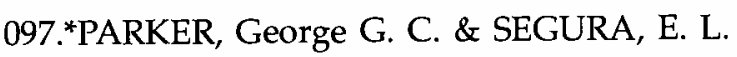
"How to get a better forecast". Harvard Business Review, 49(2):99-109, Mar./ Apr. 1971.

1969

098. *ABRAHAM, W.I. National income and economic accounting. Englewood, Prentice-Hall, $1969,232 \mathrm{p}$.

099.*EVANS, Edward C.D. Planejamento periódico e controle orçamentário. Rio de Janeiro, Tridente, 1969, 103p.
1968

100.*NELSON, G.K. Budgeting problems. New York, Ronald Press, 1968، 153p.

1966

101.*EDEY, Harold C. Business budgets and accounts: an introduction to management accounting. London, Hutchinson University, 1966, 176p.

102. *JONES, Reginald L. "Budgeting: key to planning and control. New (thinking ahead)". Harvard Business Review, 42(6):166+, Nov./Dec. 1964.

sem data

103. *FLANZER, Henrique. Orçamento Financeiro, números-índices. Rio de Janeiro, CEBC, 18p.

\section{A RAE cumpre o doloroso dever de registrar o falecimento de sua colaboradora}

\section{Madalena Sofia Mitiko Wada,}

responsável pela seção Agenda do Administrador, ocorrido em 30 de abril de 1990, em São Paulo. 